Session 1917

\title{
Simulation and Animation of Mechanical Systems to Enhance Student Learning
}

\author{
M. Hoorfar, H. Najjaran, W. L. Cleghorn \\ Mechanical and Industrial Engineering Department \\ University of Toronto
}

\begin{abstract}
Introduction
There are many applications in mechanical engineering whose analysis or design procedures not only require tedious computations but also are prone to error so that neither instructors nor students are keen to focus on the details of the subjects. They are not enthusiastic to pursue the lengthy process of the old fashioned designs although widely used in the industry. Thus, they incline to use commercial programs which are more similar to a black box. The use of educational computer programs, on the other hand, could effectively alleviate the problems because students may understand the subject and effects of many parameters involved without wasting their time for repetitive computations. It can also help them to examine the results and track the errors and see where the problems lie.
\end{abstract}

Educational computer programs are different from commercial ones in many aspects. The educational programs must have a sufficiently generic framework to deal with a large variety of possible options that may or may not be used in real applications. More precisely, commercial software often works in a limited domain whose extremes are well defined for both users and programmers, whereas educational tools should be able to satisfy curious students who naturally prefer to test the programs with irregular examples. Besides, the educational computer program must have an interesting graphical user interface including visualization and animation to motivate the users, and provide ample information and background about the application, pertinent parameters, possible errors, etc. Thus, the development of a useful educational program would be challenging for it requires a deep understanding of the subject, programming, and educational skills.

The Department of Mechanical and Industrial Engineering at the University of Toronto has been interested in the development of a series of software programs that can be used by instructors, teaching assistants, and students involved in the undergraduate curricula. The programs are primarily developed for the teaching purposes, but they can be used in distance learning, student projects, research laboratories, and educational workshops. This paper presents

"Proceedings of the 2002 American Society for Engineering Education Annual Conference \& Exposition Copyright $@$ C 2002, American Society for Engineering Education” 
two sample programs developed for two mechanical systems including mechanical vibration systems and cam and follower systems. Feedback from students who have worked with these programs has been so positive that it encourages us to consider more such applications and develop the programs, accordingly.

Mechanical Vibration Systems

This program is developed for simulation and animation of mechanical vibration of multidegree-of-freedom systems with lumped masses (Fig. 1). It is primarily used as a teaching aid for the mechanical vibration course at the undergraduate level ${ }^{1}$. However, it may be used to study the transient responses and stability of such systems. Researchers and students may gain a superior imagination of the system characteristics by interpreting the charts and observing the animation of the system. Compared to the previous works, the program is more generic and covers most cases associated with lumped mass mechanical vibration systems so that it may be considered as a useful tool for teaching and understanding of the relevant courses.

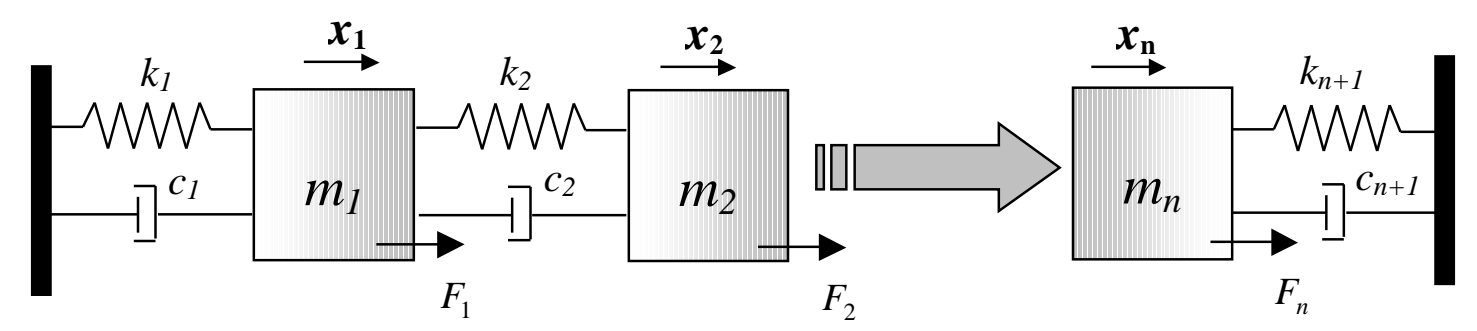

Fig. 1 Multi degree of freedom system with lumped masses, springs, and dampers

Inputs of the program are the initial conditions and parameters of the system (i.e., mass, stiffness, and damping matrices). The Runge-Kutta method is used to solve the equation of motion of damped multi-degree-of-freedom system under free and forced vibration ${ }^{2}$. The outputs of the program include the displacement and velocity of each mass as a function of time that are portrayed in the main window of the program (Fig. 2). The trajectory (i.e., velocity vs. displacement) and animation of each mass may be displayed in a separate window (Fig. 3).

The program can automatically generate commonly used physical models with lumped masses using a set of different boundary conditions. In addition, one may input their model with both dynamic and static coupling.

Five types of excitations may be applied to each mass (i.e., each degree of freedom): step, ramp, impulse, sinusoidal, and random excitations. The excitations must be of the same type amongst different masses, but they may be applied at any time and in any order. The program has been written with MATLAB ${ }^{\circledR}$ scripts which enable the students to run it in UNIX and PC platforms. 


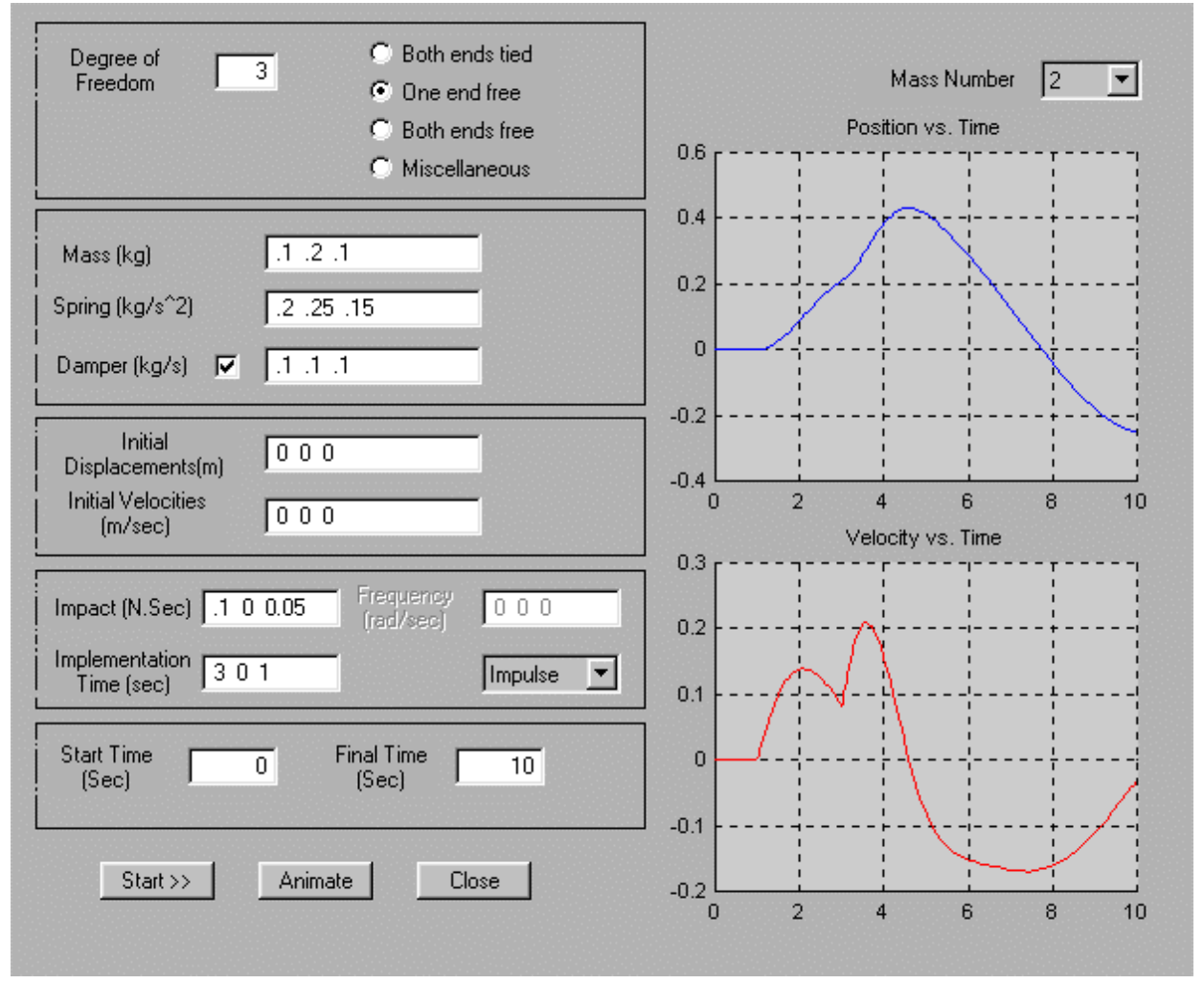

Fig. 2 Main window of the program

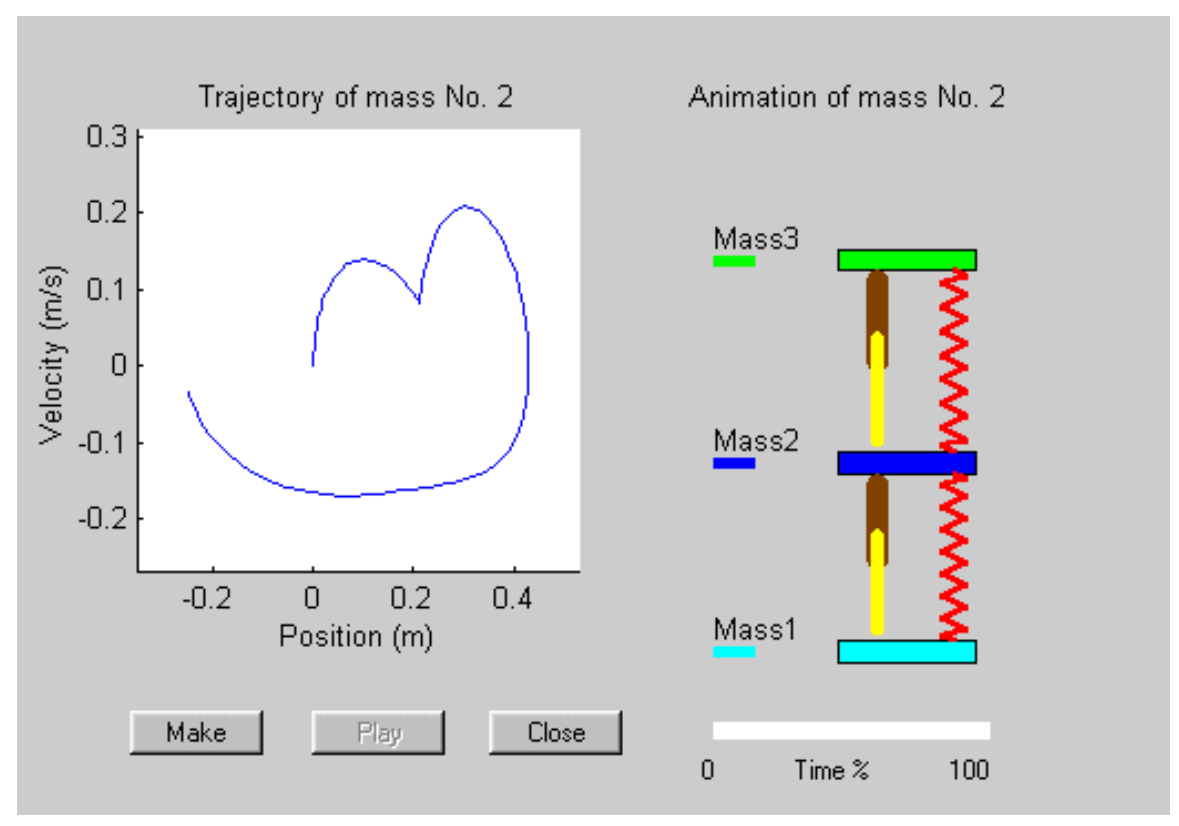

Fig. 3 Animation window of the program

"Proceedings of the 2002 American Society for Engineering Education Annual Conference \& Exposition Copyright $($ C 2002, American Society for Engineering Education" 
Cam and Follower Systems

This program is developed for learning the design of cam and follower systems, which may be offered in an undergraduate mechanisms course of the mechanical engineering curriculum. Typically, the design of the cam and follower systems is so complicated that degrades the learning of the subject. To facilitate the design procedure and improve student learning, the program provides a user-friendly toolbox that includes a large variety of cam and follower combinations whereas most previous teaching materials comprehend only limited forms of cam and follower systems ${ }^{3}$.

The program can consider pivoting and translating followers with knife edge, flat face, roller, or spherical face (Fig. 4). The type of the follower motion at each sector of the cam may be selected from a list of different motions commonly encountered. The properties of the system for a full rotation of a disc cam are computed based on the relative geometry of the cam and follower ${ }^{4}$.

Wizard dialog boxes lead the user through a step-by-step design procedure (Fig. 5). At each step, the program provides the errors, warnings, and directions associated with impossible geometries and motions. The outputs of the program (i.e., follower displacement, velocity, acceleration, and jerk) are presented in graphs and tables. Shown in Fig. 6, the schematic of the cam and follower may be shown in any desired cam orientation. The rotation and animation tools are other features of the program that help the students to obtain a better imagination of the performance of the system designed.

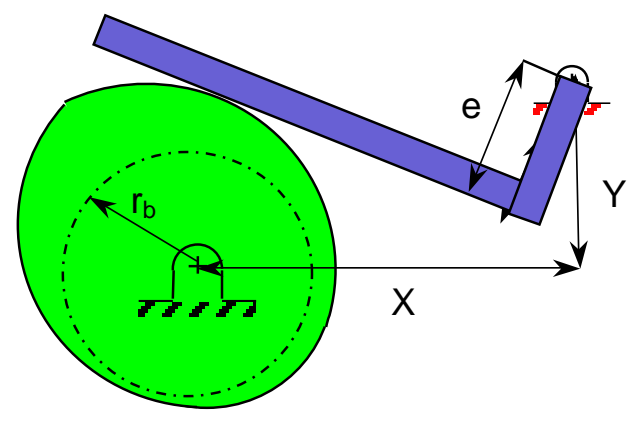

(a)

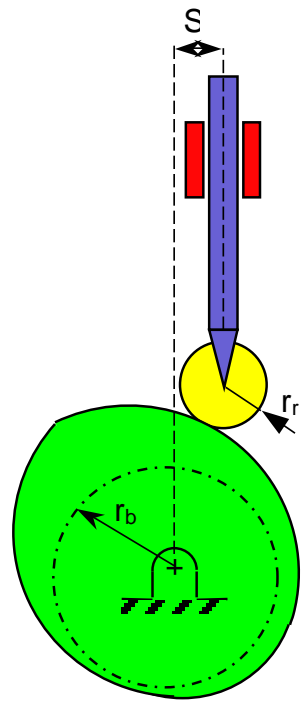

(b)

Fig. 4 Cam and Follower Systems (a) Pivoting flat face (b) Translating roller 

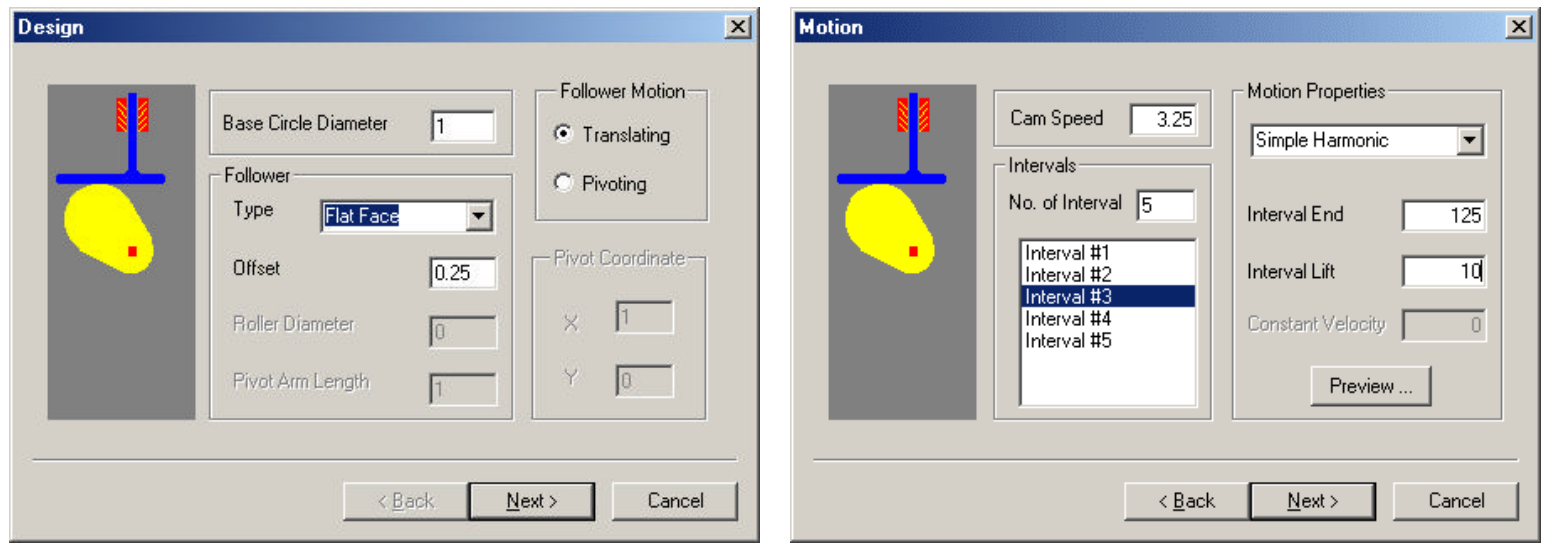

Fig. 5 Two of the wizard dialog boxes

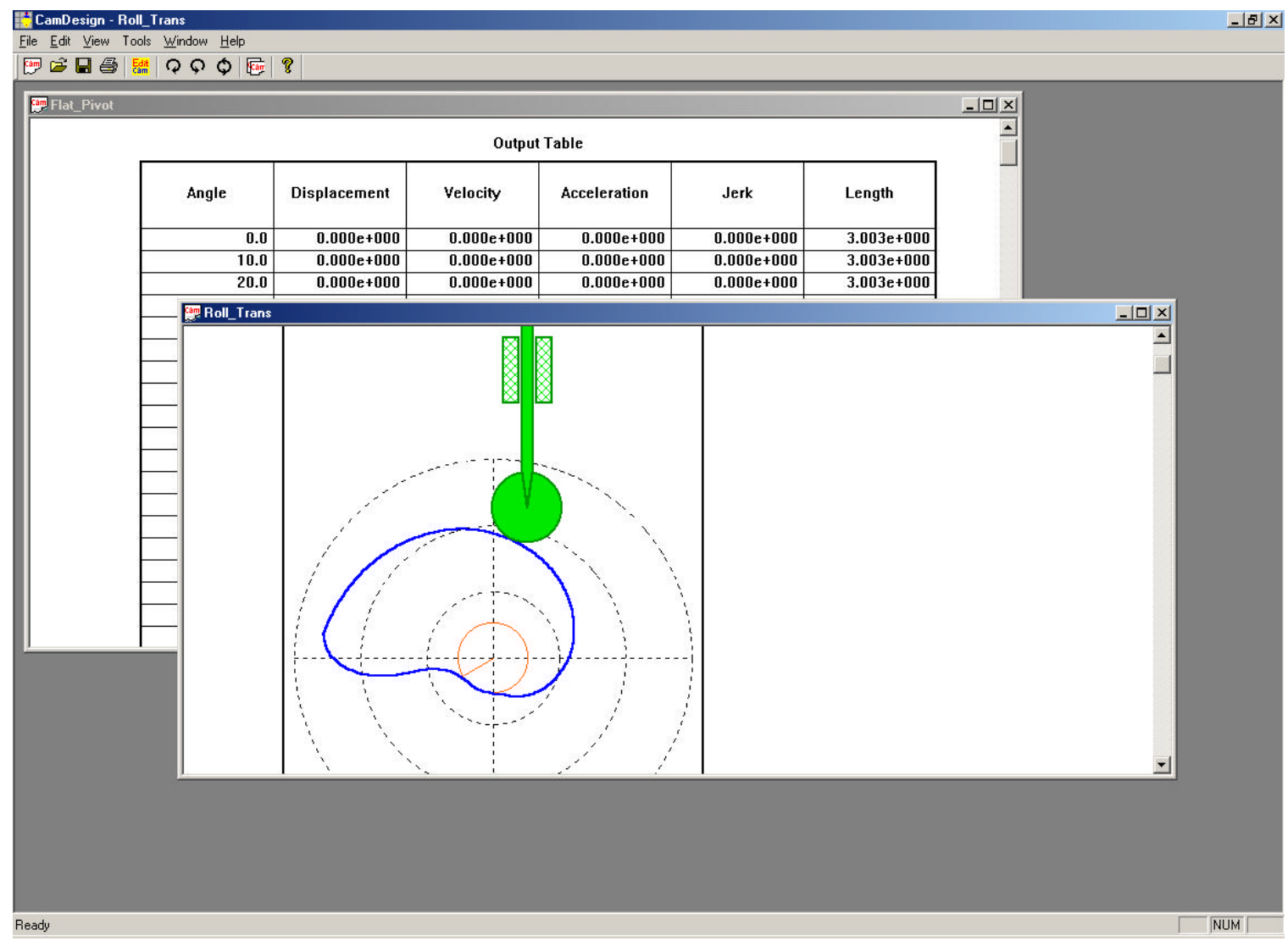

Fig. $6 \mathrm{MDI}$ interface of the program

"Proceedings of the 2002 American Society for Engineering Education Annual Conference \& Exposition Copyright $\left({ }_{0}\right.$ 2002, American Society for Engineering Education" 
The program is a multi document interface (MDI) application that presents multiple cam and follower designs simultaneously. Thus, it allows the user to study the significance of each parameter involved. In addition, the graphs and contours of selected systems may be superimposed in one window to compare the results (Fig. 7). The cam design program has been written with $\mathrm{C}++$ using the MFC library which provides a portable interface for all Microsoft Windows ${ }^{\circledR}$ platforms.

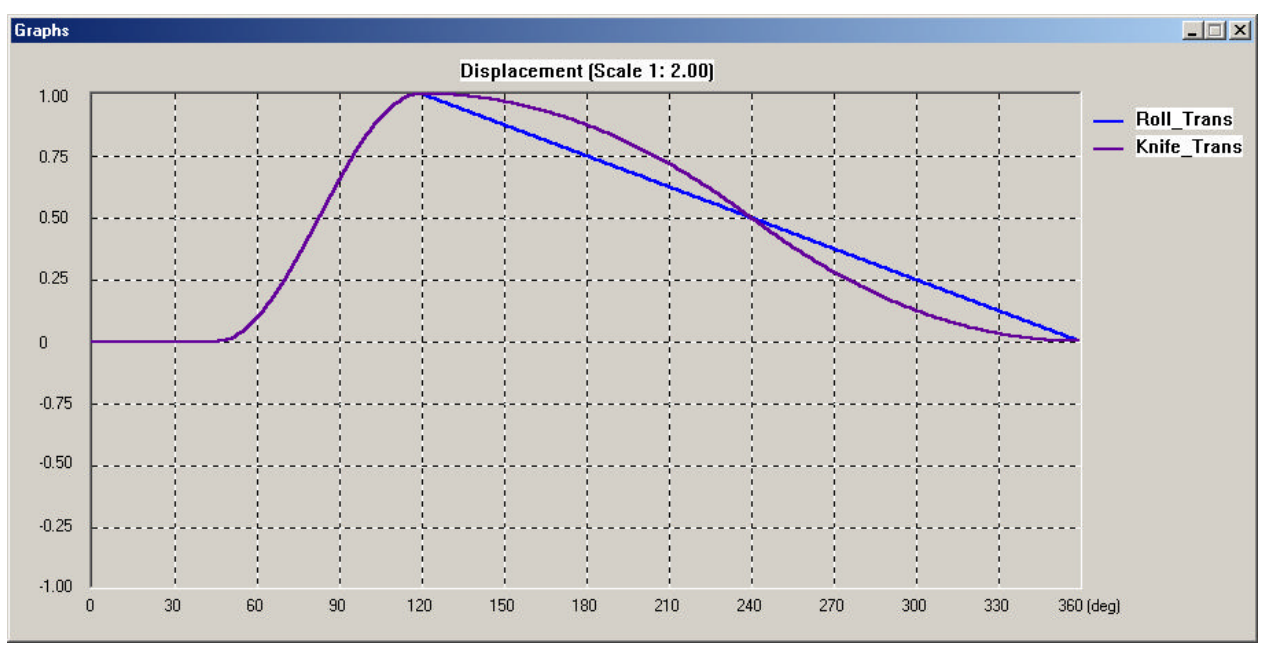

Fig. 7 Superimpose the graphs and contours of several cam and follower systems

\section{Conclusion}

The use of the computer programs has improved the learning of the students in science and engineering fields dramatically, since they can employ their time more efficiently. Nevertheless, it might have increased the reluctance of students in learning the fundamentals. To alleviate the possible drawbacks of such influences in the undergraduate level, a series of educational software programs are being developed in the mechanical and industrial engineering department of the University of Toronto that provides useful information required to enhance the student learning. In this paper, we present two software programs developed for mechanical vibration systems and cam and follower systems. The analysis and design of both systems are so time consuming that could not be scheduled in the lecture hours. Therefore, the programs have been presented to the students of the relevant courses whose feedback has been very positive.

\section{References}

[1] M. Hoorfar, H. Najjaran, W. L. Cleghorn, "Visual aid of mechanical vibration systems to enhance student learning," International Journal of Mechanical Engineering Education., (Accepted for publication)

[2] S. S. Rao, Mechanical Vibration $3^{\text {rd }}$ Edition, Addison Wesley 1995.

[3] R. L. Norton, Design of Machinery $2^{\text {nd }}$ Edition, McGraw Hill 1999. 
[4] W. L. Cleghorn, R. P. Podhorodeski, "Disc cam design using a microcomputer," International Journal of Mechanical Engineering Education Vol. 16, No. 4.

MINA HOORFAR received her M.A.Sc. degree in mechanical engineering from the University of Toronto in 2001. Currently, she is enrolled in the PhD program at the University of Toronto. Since 1999, she has been working at the Applied Surface Thermodynamics Laboratory at the University of Toronto. Her research area involves the determination of interfacial properties using digital imaging.

HOMAYOUN NAJJARAN received his M.A.Sc. degree in mechanical engineering from the University of Tehran, Iran in 1997. Currently, he is enrolled in the PhD program at the University of Toronto. Since 1999, he has been working in the Robotics and Automation Laboratory at the University of Toronto where his research is focused on using sensor fusion in the motion control of real-time autonomous mobile robots.

WILLIAM CLEGHORN graduated from the Department of Mechanical Engineering, University of Toronto. In 1980, he received his Ph.D. from the same department. He subsequently worked in industry. In 1986, he joined the Department of Mechanical Engineering, University of Toronto. Dr. Cleghorn has authored numerous publications related to vibrations. In 2001, he was appointed the Clarice Chalmers Chair of Engineering Design. 